\title{
Distinguishability of Locally Finite Trees
}

\author{
Mark E. Watkins \\ Department of Mathematics \\ Syracuse University \\ Syracuse, NY 13244-1150 \\ mewatkin@syr.edu \\ Xiangqian Zhou \\ Department of Mathematics \\ University of Mississippi \\ Oxford, MS 38677-9701 \\ xzhou@olemiss.edu
}

Submitted: Mar 30, 2006; Accepted: Mar 28, 2007; Published: Apr 4, 2007

\begin{abstract}
The distinguishing number $\Delta(X)$ of a graph $X$ is the least positive integer $n$ for which there exists a function $f: V(X) \rightarrow\{0,1,2, \cdots, n-1\}$ such that no nonidentity element of $\operatorname{Aut}(X)$ fixes (setwise) every inverse image $f^{-1}(k), k \in\{0,1,2, \cdots, n-$ $1\}$. All infinite, locally finite trees without pendant vertices are shown to be 2 distinguishable. A proof is indicated that extends 2-distinguishability to locally countable trees without pendant vertices. It is shown that every infinite, locally finite tree $T$ with finite distinguishing number contains a finite subtree $J$ such that $\Delta(J)=\Delta(T)$. Analogous results are obtained for the distinguishing chromatic number, namely the least positive integer $n$ such that the function $f$ is also a proper vertex-coloring.
\end{abstract}

\section{Introduction}

The distinguishing number $\Delta(X)$ of a graph $X$ is the least positive integer $n$ for which there exists a function $f: V(X) \rightarrow\{0,1,2, \cdots, n-1\}$ such that every element of $\operatorname{Aut}(X)$ fails to fix (setwise) at least one of the inverse images $f^{-1}(k), k \in\{0,1,2, \cdots, n-1\}$. Intuitively, $\Delta(X)$ is the least number of colors with which $V(X)$ can be colored so that no automorphism preserves all of the color classes. We call $X$ n-distinguishable if $\Delta(X) \leq n$. The notion of distinguishability is originally due to Albertson and Collins [1] and has been pursued in [6]. 
The distinguishing chromatic number of a graph $G$ was proposed by Collins and Trenk [3]. Denoted by $\chi_{\Delta}(G)$, it is the least positive integer $n$ for which there exists a function $f: V(G) \rightarrow\{0,1, \cdots, n-1\}$ such that, in addition to the above condition, also satisfies the condition that, for all $u, v \in V(G), f(u) \neq f(v)$ whenever $u$ and $v$ are adjacent.

The purpose of this paper is to determine the distinguishing number and the distinguishing chromatic number for all infinite, locally finite trees. Determination of the distinguishing number is accomplished by two main results. First we show that all infinite, locally finite trees without pendant vertices are 2-distinguishable. We then show that any locally finite tree with finite distinguishing number contains a finite subtree with the same distinguishing number. The latter result requires a two-pronged attack. First we deal with multiended trees; trees without pendant vertices fall in this category. Then we resolve the matter for one-ended trees.

Minor adaptations of these proofs yield analogous results for (1) the distinguishing chromatic number for infinite, locally finite trees and (2) the distinguishing number of locally countable trees. Our work thus extends the work of Cheng [2] and Collins and Trenk [3] for finite trees.

\section{Preliminaries}

Throughout this article, except in the concluding section $\S 6$, the symbol $T$ denotes an infinite locally finite tree, i.e., all valences are finite. A 1-valent vertex is called a pendant vertex. The usual graph metric is denoted by $\delta(\cdot, \cdot)$.

In 1964, R. Halin [4] introduced the notion of ends of graphs, an analogue to ends of groups: two rays (one-way infinite paths) in a graph belong to the same end if the intersection of some third ray with each of the given rays is infinite. For a locally finite graph $X$, the number of these equivalence classes of rays can also be defined to be the supremum of the number of infinite components of $X-Y$ as $Y$ ranges over all finite subgraphs of $X$. Every infinite, locally finite graph contains a ray (König's Theorem) and hence has at least one end. A graph is multiended if it has more than one end. In a tree, two rays are end-equivalent if and only if their intersection is a common subray. Figure 1 shows two examples of locally finite trees: the first one is called a double ray and it has two ends; the second tree has only one end.

Proposition 2.1 If a tree has no pendant vertex, then it is multiended.

Proof. Suppose that $T$ is one-ended, and let $v \in V(T)$. If $v$ is not a pendant vertex or adjacent to a pendant vertex, then $T-v$ has a component $F$ which is a nontrivial finite tree. Since nontrivial finite trees have at least two pendant vertices, at least one vertex of $F$ is not adjacent to $v$ and hence is a pendant vertex of $T$.

For each vertex $v \in V(T), H_{v}$ will denote the subtree of $T$ consisting of the union of all the finite components of $T-v$ together with the vertex $v$ and the edges joining $v$ to the union of these finite components. Since $v$ has finite valence, $H_{v}$ is finite. Such a finite subtree will be called by the botanical term epiphyte, and we say that $H_{v}$ is joined 

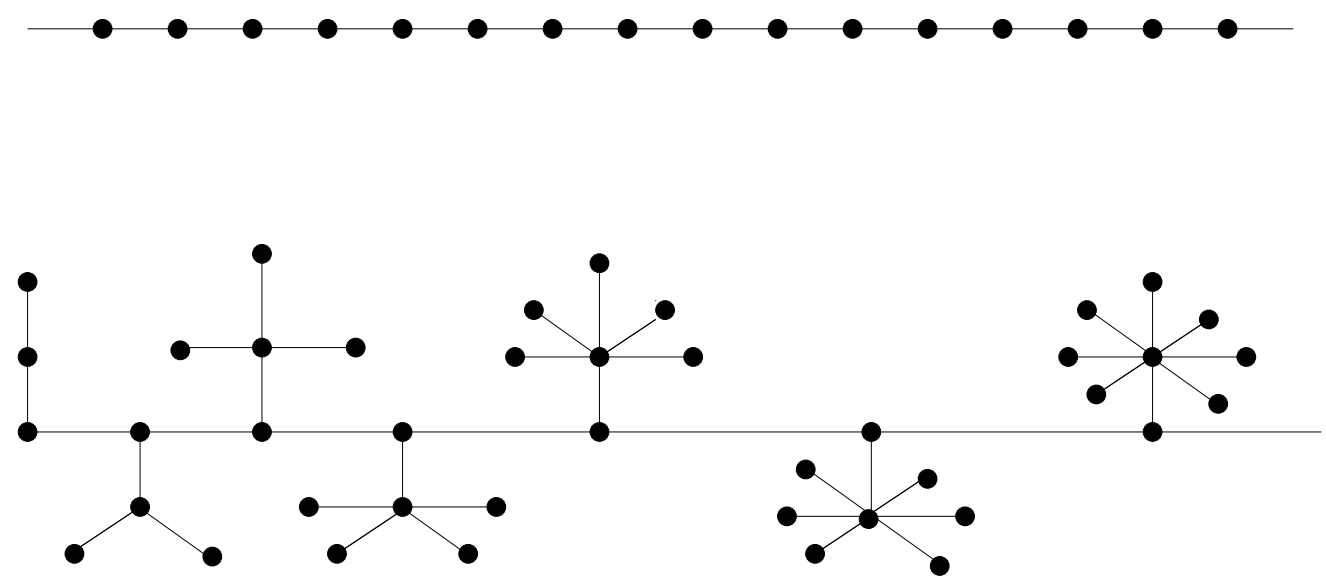

Figure 1: Examples of locally finite trees.

at $v$. For any vertex $v$ of $T$, at most one nonempty epiphyte is joined at $v$. Clearly $T$ has nonempty epiphytes if and only if $T$ has pendant vertices.

Let $W$ be the set of vertices $w \in V(T)$ such that $T-w$ has at least two infinite components. The induced subgraph $T^{c}=\langle W\rangle$ is called the core of $T$. By definition, if $T$ is one-ended if and only if $W=\emptyset$.

Suppose that $w \in W$, and let $T_{1}$ and $T_{2}$ be two infinite components of $T-w$. For $i=1,2$, let $t_{i}$ be the vertex of $T_{i}$ adjacent to $w$. Then $T-t_{1}$ also has at least two infinite components, one of which contains $T_{2}+w$. Because $T$ is locally finite, $T-t_{1}$ has only finitely many components, and so one of them must contain an infinite connected subgraph of $T_{1}-t_{1}$. With a similar argument for $t_{2}$, we conclude that every vertex in $W$ has at least two neighbors in $W$, and so the induced subgraph $\langle W\rangle$ has no pendant vertex. This implies that $W$ is infinite.

Now let $w, w^{\prime} \in W$ and let $P=\left\langle w=p_{0}, p_{1}, \ldots, p_{k}=w^{\prime}\right\rangle$ be the (unique) $w w^{\prime}$-path in $T$. Since $p_{1}$ belongs to one of (at least) two infinite components of $T-w$, the argument of the previous paragraph implies that $p_{1} \in W$. By induction, $V(P) \subset W$. Hence $\langle W\rangle$ is connected. We have shown:

Proposition 2.2 For any infinite, locally finite tree $T$, the core $T^{c}$ of $T$ is either empty or is an infinite, locally finite tree without pendant vertices. In the latter case, $T$ is multiended and $T^{c}$ is the unique maximal subtree without pendant vertices. Furthermore, $T$ has exactly one end if and only if it has an empty core.

A rooted tree is a pair $(T, z)$, where $z \in V(T)$ is its root. The distinguishing number $\Delta(T, z)$ is defined in the same way as $\Delta(T)$ except that only automorphisms in the stabilizer $\operatorname{Aut}_{z}(T)$ are considered. Thus $\Delta(T, z) \leq \Delta(T)$.

In a rooted tree $(T, z)$, the level of any vertex $v$ is its distance from the root $z$, the root itself being at level zero. If $v \neq z$, then the parent of $v$ is the neighbor of $v$ on the $z v$-path in $T$. If $v$ is the parent of $w$, then $w$ is an offspring of $v$. Vertices having a common parent are siblings. (These terms are borrowed from [3].) 


\section{Multiended Trees}

Theorem 3.1 Every locally finite infinite tree without pendant vertices is 2-distinguishable.

Proof. Let $(T, z)$ be a rooted tree, where $T$ has no pendant vertices. For $\ell \geq 0$, let $S_{\ell}$ denote the $\ell$-sphere about $z$, i.e., the set of vertices at level $\ell$. Since $T$ has no pendant vertices, clearly $\left|S_{\ell}\right| \leq\left|S_{\ell+1}\right|$ for all $\ell \geq 0$.

We establish a lexicographic ordering of the set $V(T)$. The ordering is first by level: $z$ at level 0 is the first element, and then the elements of $S_{1}$ are ordered arbitrarily. Assuming that $S_{\ell}$ has been ordered, if $y, y^{\prime} \in S_{\ell}$ and $y^{\prime}$ is the successor of $y$, then the offsprings of $y$ are ordered arbitrarily followed immediately by the offsprings of $y^{\prime}$ also ordered arbitrarily. In this manner, the set $S_{\ell+1}$ is ordered.

Let $\beta_{1}$ denote the binary sequence

$$
\beta_{1}:=1010^{2} 10^{3} 1 \cdots 10^{k} 10^{k+1} 1 \cdots,
$$

where $0^{k}$ indicates $k$ consecutive 0 s. Let $\beta_{m}$ be the tail of $\beta_{1}$ beginning with the $m^{\text {th }} 1$; thus

$$
\beta_{m}=10^{m} 10^{m+1} 10^{m+2} 1 \cdots . \quad(m \in \mathbb{N}) .
$$

We now define a coloring $f: V(T) \rightarrow\{0,1\}$. To begin, let $f(z)=1$. Suppose that $S_{1}=\left\{y_{1}, \cdots, y_{r}\right\}$, where the indices are consistent with above-described ordering. For $m=1, \ldots, r$, let $R_{m}$ denote the ray emanating from $y_{m}$ that proceeds next to $y_{m}$ 's first offspring $y_{m 1}$, then to that offspring's first offspring, ad infinitum. (Such a ray exists because there are no pendant vertices.) Use $\beta_{m}$ to color this ray: the $j$ th vertex on the ray $R_{m}$ is assigned the $j$ th digit of $\beta_{m}$.

We next complete the coloring of $S_{2}$, noting that the first offspring $y_{m 1}$ of each $y_{m} \in S_{1}$ has been colored by the first 0 of $\beta_{m}(m=1, \ldots, r)$. If $y_{1}$ has a second offspring $y_{12}$, let $R_{12}$ be the ray emanating from $y_{12}$ that proceeds to $y_{12}$ 's first offspring, then to that offspring's first offspring, ad infinitum, and use $\beta_{r+1}$ to color the vertices of $R_{12}$ in the same manner. Thus each of the $s$ siblings of $y_{11}$ is the initial vertex of a ray colored via $\beta_{r+1}, \ldots, \beta_{r+s}$, respectively. We then move on the second offspring of $y_{2}$.

In general, as the vertices are encountered in lexicographic order, they become the initial vertices of rays colored via the next available sequence $\beta_{m}$ with two exceptions to this rule:

1. Vertices already colored (because they lie on a ray defined at a lower level) are skipped over;

2. Suppose that a vertex $x^{\prime}$ is the second offspring of a parent $v$ which is already colored 0 , while the first offspring $x$ of $v$ is already colored 1. Suppose further that the number of consecutive vertices following $x$ on the already-colored ray through $v$ and $x$ that have been colored 0 is $n$, that the next unused sequence is $\beta_{m}$, and that $m \leq n$. In this case skip over the sequences $\beta_{m}, \beta_{m+1}, \ldots, \beta_{n}$, and let the ray emanating from $x^{\prime}$ be colored via $\beta_{n+1}$. 
(This second exception is not vacuous, as illustrated in Figure 2, in particular if there are many 2 -valent vertices closer to the root. In the figure, the vertices assigned to 0 are colored white, and those assigned to 1 are colored black. Note that $\beta_{4}$ and $\beta_{6}$ must be skipped over.)

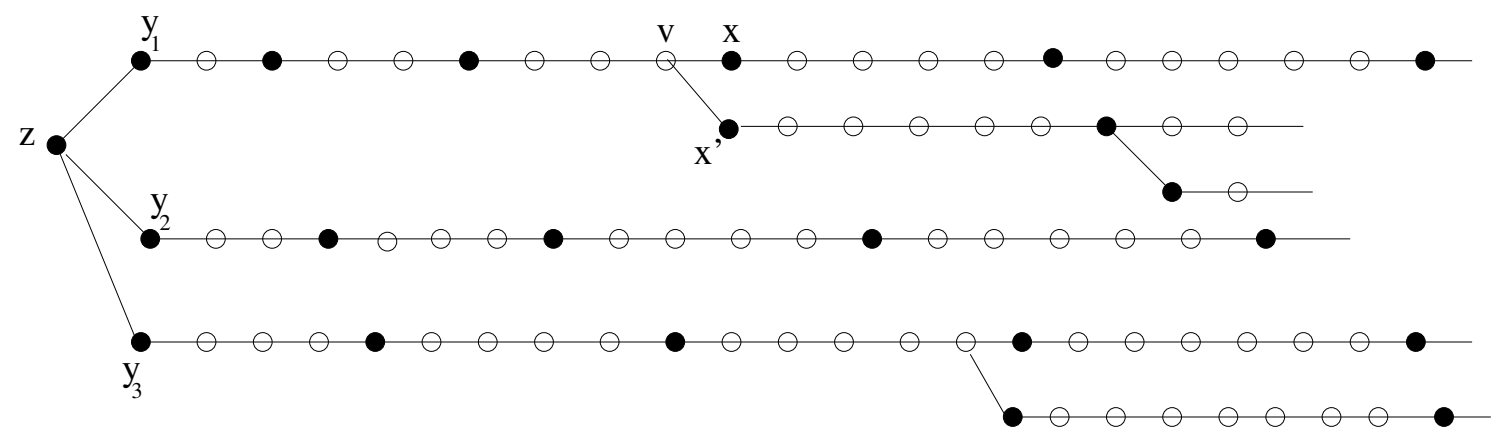

Figure 2: the second exception.

We now show that no automorphism of $T$ fixes the color class $f^{-1}(1)$, but first we make some observations.

1. The root $z$ is the only vertex colored 1 , all of whose neighbors are also colored 1 . Hence $z$ is a fixed point of any automorphism fixing the set $f^{-1}(1)$.

2. If a vertex other than $z$ is colored 1, then exactly one of its offsprings, namely its first offspring, is colored 0 .

3. If a vertex is colored 0 , then at most one of its offsprings is colored 0 .

Let $\varphi$ be a non-identity element of $\operatorname{Aut}(T)$ and suppose that $\varphi$ fixes $f^{-1}(1)$ setwise. Noting that, if $\varphi$ fixes a vertex $v$, then it fixes each vertex of the (unique) $z v$-path, pick the largest $\ell$ such that $\varphi$ fixes $S_{\ell}$ pointwise. Let $v \in S_{\ell}$. It suffices to prove that $\varphi$ fixes each of $v$ 's offsprings.

If $v$ has only one offspring, the conclusion is obvious, so suppose that $x$ and $x^{\prime}$ are offsprings of $v$ with $x$ immediately preceding $x^{\prime}$ is the lexicographic order. In the light of observations 2 and 3 above, we may assume that $f(x)=f\left(x^{\prime}\right)=1$ and that each of $x$ and $x^{\prime}$ has a unique offspring colored 0 . Let $n$ be the length of the longest path starting at $x$ such that all its vertices except $x$ are colored 0 . Then there are exactly two possibilities:

Case 1: $x$ is $v$ 's first offspring. In this case the second exception applies. The sequence $\beta_{n+1}$ was used to color a ray emanating from $x^{\prime}$, and so $n+1$ is the length of the longest path starting at $x^{\prime}$ such that all its vertices except $x^{\prime}$ are colored 0 . Not only must $f$ not map $x$ onto $x^{\prime}$, but a fortiori $f$ cannot map $x$ onto any of its siblings.

Case 2: $x$ is not $v$ 's first offspring. In this case the "generic" coloring rule applies: $x$ and $x^{\prime}$ are initial vertices of rays colored via $\beta_{n}$ and $\beta_{n+1}$, respectively, and the argument proceeds as in Case 1. 
Remark. The 2-coloring described in the foregoing theorem has an additional property. Not only is there no automorphism that fixes each color class (setwise), but we can require as well that no automorphism interchange the two color classes. If $T$ is simply a double ray, then one easily verifies that the 2-coloring given in the proof of the theorem yields the desired result. Otherwise, one may select any vertex of valence $\geq 3$ to be the root. The root is then the only vertex of valence $\geq 3$ to receive the same color as all of its neighbors.

Theorem 3.2 Let $T$ be an infinite, locally finite, multiended tree. Unless $T$ is asymmetric,

$$
\Delta(T)=\sup \left\{2,\left\{\Delta\left(H_{v}, v\right): v \in V\left(T^{c}\right)\right\}\right\} .
$$

Proof. If all epiphytes of $T$ are empty, i.e., if $T=T^{c}$, then $\Delta(T)=1$ if $T$ is asymmetric. Otherwise $\Delta(T)=2$ by Theorem 3.1.

The restriction to $T^{c}$ of any automorphism of $T$ is clearly an automorphism of $T^{c}$. Let $v \in V\left(T^{c}\right)$, and consider its orbit $O(v)=\{\varphi(v): \varphi \in \operatorname{Aut}(T)\}$. Let $A(v)$ denote the permutation group on $O(v)$ induced by $\operatorname{Aut}(T)$. For every vertex $w \in O(v), H_{w}$ is isomorphic to $H_{v}$. Thus $\operatorname{Aut}(T)$ contains a wreath product $\operatorname{Aut}_{v}\left(H_{v}\right)$ \& $A(v)$. (Clearly if $H_{v}$ is empty, then the first factor of the wreath product is trivial.) Aut $(T)$ is itself some sort of product over the orbits of $\operatorname{Aut}(T)$ of these wreath products. If the color classes of a coloring of $V(T)$ are preserved by none of these wreath products, then they will also be preserved by no nonidentity element of $\operatorname{Aut}(T)$. We describe such a coloring.

First let $T^{c}$ be 2-colored exactly as in the proof of Theorem 3.1. If $\varphi \in \operatorname{Aut}(T)$ fixes the two color classes in $V\left(T^{c}\right)$, then $\varphi$ is the identity when restricted to $T^{c}$. Furthermore, the restriction $\varphi_{\mid H_{v}}$ belongs to the stabilizer $A_{v}\left(H_{v}\right)$. Now let $V\left(H_{v}\right)$ be colored with the least number of colors so that no automorphism in $\operatorname{Aut}_{v}\left(H_{v}\right)$ preserves all of the color classes. It follows that $\Delta(T) \geq \Delta\left(H_{v}, v\right)$ for any vertex $v$ of $T^{c}$.

For each vertex $v \in V\left(T^{c}\right)$, a permutation of the colors of $H_{v}$ is possible so that, in that epiphyte, the vertex $v$ is assigned the same color 0 or 1 that $v$ was assigned in $T^{c}$. This completes the coloring of $V(T)$. It follows that, if there exists some $n$ such that $2 \leq \Delta\left(H_{v}, v\right) \leq n$ whenever $H_{v}$ is not empty, then $\Delta(T) \leq n$, completing the proof.

Of the two graphs in Figure 1, the double ray has distinguishing number 2 by Theorem 3.1. The second graph has infinite distinguishing number because the distinguishing numbers of its epiphytes are not bounded.

Corollary 3.3 Let $T$ be an infinite, locally finite, multiended tree. If $\Delta(T)=n<\infty$, then $T$ contains a finite subtree $J$ such that $\Delta(J)=n$.

Proof. By Theorem 3.1, if all epiphytes of $T$ are empty, then $\Delta(T)=1$ or 2, in which case we note that $T$ contains copies of $K_{1}$ and $K_{2}$. Otherwise, by Theorem 3.2, $T$ has an epiphyte $H_{v}$ such that $v \in V\left(T^{c}\right)$ and $\Delta(T)=\Delta\left(H_{v}, v\right)=n$. If $\operatorname{Aut}\left(H_{v}\right)=\operatorname{Aut}_{v}\left(H_{v}\right)$, then we're done. If $\operatorname{Aut}\left(H_{v}\right) \neq \operatorname{Aut}_{v}\left(H_{v}\right)$, then rather than attempt to destroy any automorphism of $H_{v}$ that does not fix $v$, we consider instead the finite subgraph $J$ obtained by adjoining to $H_{v}$ a path in $T^{c}$ beginning at $v$ of length $|V(H)|+1$. (Such a path exists by Proposition 2.2.) Then $\Delta(J)=\Delta\left(H_{v}, v\right)=n$ as required. 


\section{One-Ended Locally Finite Trees}

One-ended trees have properties that require a different approach. Unlike in multiended trees, the intersection of any two rays is a common subray. By a result of Halin ([5], Theorem 7), the automorphism group of a one-ended tree $T$ admits no translation. Thus every automorphism of $T$ admits only orbits of finite length, although an automorphism may have infinitely many orbits and there need be no upper bound to their lengths. In fact, with an empty core, one-ended trees are nothing but an infinite union of epiphytes within epiphytes.

Lemma 4.1 Every automorphism of a one-ended locally finite tree has a fixed point.

Proof. Suppose that $T$ is a one-ended locally finite tree, let $\alpha \in \operatorname{Aut}(T)$, and suppose that $\alpha$ has no fixed point.

Claim 1: If $\alpha$ fixes a path $P$ in $T$, then $\alpha$ fixes $P$ pointwise.

If $\alpha$ fixes $P$ setwise but not pointwise, then $\alpha$ swaps the two terminal vertices of $P$. Since $\alpha$ has no fixed point, $P$ has odd length and $\alpha$ must fix a unique edge $e=[u, v]$ on $P$. Since $T$ is one-ended, $T-e$ has exactly one infinite component, and that component must contain exactly one of $u$ and $v$. Although $\alpha$ fixes $e, \alpha$ cannot then swap $u$ and $v$, a contradiction, proving Claim 1.

Arbitrarily choose $x \in V(T)$. Since $\alpha(x) \neq x$, let $P$ denote the unique path from $x$ to $\alpha(x)$. Since $\alpha$ admits only orbits of finite length, there exists a least integer $n>1$ such that $\alpha^{n}(x)=x$. If $n=2$, then $\alpha$ would fix $P$ setwise but not pointwise, contrary to Claim 1. Hence $n \geq 3$.

Let $z$ be the vertex of $P \cap \alpha(P)$ that is nearest to $x$. Then $\alpha(z)$ is the vertex in $\alpha(P) \cap \alpha^{2}(P)$ that is nearest to $\alpha(x)$. By assumption, $\alpha(z) \neq z$.

Claim 2: $\alpha(z) \notin V(P)$.

Suppose $\alpha(z) \in V(P)$. Then $\alpha(z)$ is on the subpath of $P$ joining $\alpha(x)$ and $z$. Since $z \in \alpha(P)$, there exits $u \in V(P)$ such that $\alpha(u)=z$. Since $\delta(u, z)=\delta(\alpha(u), \alpha(z))=$ $\delta(z, \alpha(z))$, either $u=\alpha(z)$, in which case $\alpha$ would fix the path from $z$ to $\alpha(z)$ contrary to Claim 1 , or $u$ is on the subpath of $P$ joining $x$ and $z$. This, too, is a contradiction since $\delta(x, u)=\delta(\alpha(x), z)>\delta(\alpha(x), \alpha(z))=\delta(x, z)$, yielding Claim 2.

Now let $Q$ be the path from $z$ to $\alpha(z)$. It is easily checked that $\bigcup_{i=0}^{n-1} \alpha^{i}(Q)$ is a nontrivial cycle, contrary to the assumption that $T$ is a tree.

Since for every vertex $z$ of a one-ended tree, there exists a unique ray emanating from $z$, we immediately have the following result.

Corollary 4.2 Each automorphism of a one-ended, locally finite tree fixes some ray pointwise.

For any graph $X$, an $m$-coloring of $X$ is called destructive if no element of $\operatorname{Aut}(G)$ fixes setwise every color class.

Theorem 4.3 Let $T$ be a one-ended locally finite tree, and let $n$ be a positive integer. $\Delta(T)=n$ if and only if $\max \left\{\Delta\left(H_{z}, z\right): z \in V(T)\right\}$ exists and equals $n$. 
Proof. Assume that $\Delta(T)=n$. For any $z \in V(T), \operatorname{Aut}_{z}\left(H_{z}\right)$ is the restriction to $H_{z}$ of $\operatorname{Aut}_{z}(T)$. Hence $\Delta\left(H_{z}, z\right) \leq \Delta(T, z) \leq \Delta(T)=n$. Let $m:=\max \left\{\Delta\left(H_{z}, z\right): z \in V(T)\right\}$ (which clearly exists), and so $m \leq n$.

We may choose an epiphyte $H_{0}$ joined at $z_{0}$ such that $\Delta\left(H_{0}, z_{0}\right)=m$. Let $R$ with $V(R)=\left\{z_{0}, z_{1}, z_{2}, \ldots\right\}$ be the ray emanating from $z_{0}$ and let $H_{i}$ denote the epiphyte joined at $z_{i}$. Since $T$ is one-ended, $H_{i}$ is a subtree of $H_{i+1}$ and $\operatorname{Aut}_{z_{i}}\left(H_{i}\right)$ is isomorphic to a subgroup of $\operatorname{Aut}_{z_{i+1}}\left(H_{i+1}\right)$ for all $i \geq 0$. Thus $m \leq \Delta\left(H_{i}, z_{i}\right) \leq \Delta\left(H_{i+1}, z_{i+1}\right) \leq m$, and so equality holds for all $i \geq 0$.

We now define inductively a coloring $f: V(T) \rightarrow\{0,1, \ldots, m-1\}$. First choose any destructive $m$-coloring of $\left(H_{0}, z_{0}\right)$. This is possible since $\Delta\left(H_{0}, z_{0}\right)=m$; let $w_{0}$ be the number of distinct destructive $m$-colorings of $\left(H_{0}, z_{0}\right)$. Since $\Delta\left(H_{1}, z_{1}\right)=m$, in the epiphyte $H_{1}$, there are (including $\left.H_{0}\right)$ at most $w_{0}$ copies of $\left(H_{0}, z_{0}\right)$ attached to $z_{1}$ by an edge between $z_{1}$ and the root of the copy; otherwise $\left(H_{1}, z_{1}\right)$ would have no destructive $m$-coloring. Therefore, any destructive $m$-coloring of $\left(H_{0}, z_{0}\right)$ can be extended to a destructive $m$-coloring of $\left(H_{1}, z_{1}\right)$. Choose such a destructive $m$-coloring of $\left(H_{1}, z_{1}\right)$ and proceed inductively to $\left(H_{2}, z_{2}\right)$. Thus we have defined an $m$-coloring $f$ of $T$ such that for any integer $i \geq 0$, the restriction of $\left.f\right|_{H_{i}}$ is a destructive $m$-coloring of $\left(H_{i}, z_{i}\right)$. We now show that $f$ is a destructive coloring of $T$.

Suppose $\alpha \in \operatorname{Aut}(T)$ is not the identity. By Corollary 4.2, there exists a ray $R^{\prime}$ fixed pointwise by $\alpha$. Choose $k$ large enough such that $z_{k} \in V\left(R \cap R^{\prime}\right)$ and the restriction of $\alpha$ to $\left(H_{k}, z_{k}\right)$ is not the identity. Since $\left.f\right|_{\left(H_{k}, z_{k}\right)}$ is a destructive coloring of $\left(H_{k}, z_{k}\right)$, and $\left.\alpha\right|_{\left(H_{k}, z_{k}\right)}$ is not the identity of $\operatorname{Aut}\left(H_{k}, z_{k}\right)$, there exists a color class, say $f_{\mid H_{k}}^{-1}(j)$, of $H_{k}$, that is not fixed by $\alpha$. It follows that $\alpha$ does not fix the color class $f^{-1}(j)$ of $T$. Hence $m \geq n$.

Combining Corollary 3.3 and Theorem 4.3, we obtain

Corollary 4.4 Every infinite, locally finite tree with finite distinguishing number contains a finite subtree with the same distinguishing number.

\section{Distinguishing Chromatic Numbers of Locally Fi- nite Trees}

Every nontrivial tree $T$ admits a unique bipartition of its vertex set, thereby determining a proper 2-coloring (of the vertex set) up to interchanging the colors of its two color classes. Thus $\chi_{\Delta}(T) \geq 2$. Since every automorphism of $T$ either fixes or interchanges these color classes, the set of automorphisms that fix each class forms a subgroup of $\operatorname{Aut}(T)$ of index 2.

Theorem 5.1 Let $T$ be an infinite locally finite tree without pendant vertices. Then $2 \leq \chi_{\Delta}(T) \leq 3$. Moreover, $\chi_{\Delta}(T)=2$ if and only if either of the following holds:

(1) $T$ is asymmetric; or 
(2) $\operatorname{Aut}(T)=\{1, \sigma\}$ is a group of order 2 and $\sigma$ interchanges the two color classes of the proper 2-coloring of $T$.

Proof. The proof that $\chi_{\Delta}(T) \leq 3$ is essentially that of Theorem 3.1: replace each instance of $\beta_{m}$ by the ternary sequence $\gamma_{m}=1(02)^{m} 1(02)^{m+1} 1(02)^{m+2} 1 \cdots$, where $(02)^{1} \equiv$ 02 and $(02)^{k+1} \equiv 02(02)^{k}$ for $k \geq 1$.

Now assume that $\chi_{\Delta}(T)=2$. Hence the subgroup of $\operatorname{Aut}(T)$ that fixes the color classes is trivial. It follows that $|\operatorname{Aut}(T)|=1$ or 2 . The former corresponds to $T$ being asymmetric. The latter corresponds to the existence of a unique automorphism that interchanges the color classes. (This argument is similar to the proof of Theorem 3.1 in [3].)

The next two propositions are analogues of Theorems 3.2 and 4.3, respectively.

Proposition 5.2 Let $T$ be a locally finite tree with a nonempty core and pendant vertices. Then

$$
\chi_{\Delta}(T)=\sup \left\{\chi_{\Delta}\left(T^{c}\right),\left\{\chi_{\Delta}\left(H_{v}, v\right): v \in V\left(T^{c}\right)\right\}\right\}
$$

Proposition 5.3 Let $T$ be an infinite locally finite one-ended tree. Then

$$
\chi_{\Delta}(T)=\sup \left\{\chi_{\Delta}\left(H_{v}, v\right): v \in V(T)\right\}
$$

\section{Locally Countable Trees}

A reasonable direction for generalization of the foregoing work would be to drop the assumption of local finiteness, or at least to bound the valences by the first infinite cardinal $\aleph_{0}$. In this situation, epiphytes need not be finite!

On the other hand, the statement of Theorem 3.1 is extendable: Every multiended, locally countable tree without pendant vertices is 2-distinguishable. However, the lexicographic ordering of the vertices used in the proof is superseded by a Cantor diagonal ordering for the purpose of selecting initial vertices of rays to be colored via the sequences $\beta_{m}$. The ordering is done in the following way. Suppose that $v$ is the $k$ th vertex in $S_{\ell}$ according to the lexicographic ordering. If $\left|S_{\ell-1}\right|>k$, then the successor of $v$ is the $(k+1)$ st vertex of $S_{\ell-1}$. Otherwise, $v$ 's successor is the first vertex of $S_{\ell+k+1}$. Certainly the list of "exceptions" becomes more complicated. The authors graciously leave this task to the reader.

\section{References}

[1] M. O. Albertson and K. L. Collins, Symmetry breaking in graphs, Electron. J. Combin. 3 (1996), \#R18.

[2] C. T. Cheng, On computing the distinguishing numbers of trees and forests, Electron. J. Combin. 13 (2006), \#R11. 
[3] K. L. Collins and A. N. Trenk, The distinguishing chromatic number, Electron. J. Combin. 13 (2006) \#R16, 19 pp.

[4] R. Halin, Über unendliche Wege in Graphen, Math. Ann. 157 (1964), 125-137.

[5] R. Halin, Automorphisms and Endomorphisms of Infinite Locally Finite Graphs, Abh. Math. Sem. Univ. Hamburg (1973), 251-283.

[6] S. Klavžar, T.-L. Wong, and X. Zhu, Distinguishing labellings of group action on vector spaces and graphs, J. Algebra 303 (2006), 626-641. 